\title{
Robust Multi-loop Airborne SLAM in Unknown Wind Environments
}

\author{
Jonghyuk Kim \\ Department of Engineering \\ Australian National University, Australia \\ Email: jonghyuk.kim@anu.edu.au
}

\author{
Salah Sukkarieh \\ ARC Centre for Autonomous Systems \\ University of Sydney, Australia \\ Email: salah@acfr.usyd.edu.au
}

\begin{abstract}
This paper presents a robust multi-loop airborne SLAM structure which also augments wind information. The air velocity observation from an air data system can be used to estimate the error of the on-board Inertial Navigation System (INS). However, due to a priori unknown wind velocity, it cannot directly be used for this purpose. This can be tackled by augmenting the unknown wind velocity into the state vector of SLAM, simultaneously estimating INS, map and wind. This paper proposes a multi-loop SLAM architecture, where the periodic velocity-level SLAM loop limits the INS errors of the velocity effectively, and the aperiodic position-level SLAM loop bounds the overall position error growth. This can significantly increase the consistency of airborne SLAM at the time of loop closure. Simulation results show that the unknown wind vector can be estimated consistently and the robustness of airborne SLAM improves significantly.
\end{abstract}

\section{INTRODUCTION}

SLAM has been demonstrated and verified in many robotic applications in indoor, land and underwater environments [1][2][3]. Airborne SLAM expands on this concept to 6-DoF (Degrees of Freedom) platforms. Due to the 6-DoF nature, conventional dead reckoning sensors cannot be applied here. Instead, an Inertial Measurement Unit (IMU) or Inertial Navigation System (INS) needs to be incorporated as demonstrated in [4]. Using INS however, introduces additional computational complexity to SLAM, since the INS has to run with a sufficiently high-update rate to track vehicle motions and to suppress vibrations. An efficient form of airborne SLAM based on a error dynamic model has thus been introduced improving the efficiency and complexity [5].

The current obstacle in implementing robust airborne SLAM is the statistical inconsistencies which arise during loop closure after extended flight times. The limited field of view of the airborne sensor and the sparse nature of the ground features can inevitably increase the vehicle's uncertainties before revisiting any previous feature. The position error growth rate of unaided INS is typically a cubic function of flight time. This means that if feature aiding is not effective, then inertial SLAM will develop large uncertainties exceeding acceptable levels for linearisation. Moreover, any over-confident correction in INS attitude can lead to a failure in inertial based SLAM. This is because even a small attitude error will misinterpret the gravitational acceleration as the dynamic acceleration, resulting in rapid divergence in the position and velocity.
This, in turn, will invalidate and reject successive observations afterward.

This problem can be tackled by using a higher quality IMU, nonlinear filtering techniques, or stabilising the attitude by adding additional information. If a high-quality IMU is an available option, it should be selected in such a way that the INS error remains within a reasonable bound before loop closure. The INS error growth, however, depends on the availability of the ground features which is difficult to predict in advance. Additionally, many high-quality inertial sensors may not be suitable for most robotic platforms which are typically payload limited. Nonlinear filtering, such as the particle filter, can reduce the chance of divergence during loop closure. It was observed that it can improve the performance compared to the standard Kalman filter, but still showed divergences during loop closure, but more importantly suffers from the dimensionality problem. The effectiveness of this approach needs further investigation in airborne SLAM applications.

The stability of the attitude estimate is of importance for reliable SLAM loop closure. Hence if there exists any constant stream of information to limit the attitude uncertainty, it will improve the robustness of airborne SLAM. In many airborne applications, the air velocity observation is directly available from the onboard air-data system, which comprises a pitot tube, alpha/beta vanes, and data acquisition system. It provides airspeed, angle of attack and side-slip angle, which are important parameters in maintaining aircraft stability. The air velocity, which is a relative velocity with respect to the surrounding wind mass, can be constructed from these observations. The wind velocity, however, is typically unknown a-priori and thus the air observations cannot directly be used for inertial aiding. One solution, proposed in this paper, is to augment this unknown wind velocity into the existing SLAM state vector, running SLAM both in position and velocity levels. This method adds extra information to the system, but it does not require further physical hardware but only simple modification to software. In addition, the air-data system is self-contained, and thus the autonomy of airborne SLAM can be maintained.

Fig. 1 describes this multi-loop SLAM architecture within the indirect framework (that is, based on error dynamic model). The high frequency INS loop $(\geq 100 \mathrm{~Hz})$ delivers real-time vehicle states to the external guidance and control logic. 


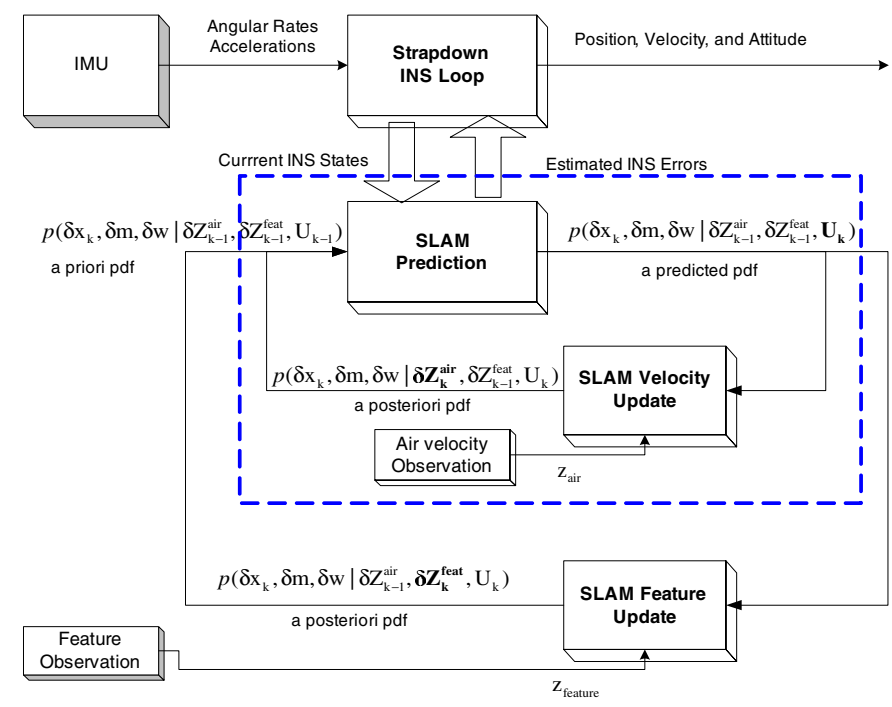

Fig. 1. Multi-loop architecture of airborne SLAM: the inner-periodic SLAM loop (within a broken box) is for velocity/attitude stabilisation and the outeraperiodic SLAM loop is for feature observation update.

The medium frequency loop with air-data observations $(\geq$ $10 \mathrm{~Hz}$ ) estimates the wind velocity and stabilises the INS velocity/attitude, forming a velocity level SLAM loop. The attitude error is estimated through the correlations between the velocity and attitude errors. It however does not provide any information to position, and thus the position level SLAM loop is used with aperiodic feature observations. The indirect formulation makes an efficient means for this multi-loop configuration.

This paper is organised as follows: Section 2 will briefly present the high speed inertial navigation loop. Section 3 will provide a Bayesian formulation of the indirect airborne SLAM with unknown wind velocity augmentation. Section 4 will describe the Kalman Filter implementation with the details of the air-data observation. Sections 5 and 6 will provide the results of simulation analysis for the Brumby MkIII UAV platform. Section 8 will conclude with future work.

\section{INERTIAL NAVIGATION LOOP}

In the indirect airborne SLAM architecture, a deterministic INS loop predicts the high-dynamic vehicle states from IMU measurements in real-time. The SLAM filter resides outside this loop, performing a complementary aiding role. The quaternion-based strapdown INS algorithm is used with an assumption of flat and non-rotating Earth-frame. This is a reasonable assumption for the applications with a low quality inertial, low speed, and short coverage (typically within $100 \mathrm{Km}$ ). The discrete equation can be written as

$\left[\begin{array}{l}\mathbf{p}^{n}(k+1) \\ \mathbf{v}^{n}(k+1) \\ \mathbf{q}^{n}(k+1)\end{array}\right]=\left[\begin{array}{c}\mathbf{p}^{n}(k)+\mathbf{v}^{n}(k) \triangle t \\ \mathbf{v}^{n}(k)+\left[\left(\mathbf{C}_{b}^{n}\left(\mathbf{q}^{n}(k)\right) \overline{\mathbf{f}}^{b}(k)\right)+\mathbf{g}^{n}\right] \triangle t \\ \mathbf{q}^{n}(k) \otimes \triangle \mathbf{q}^{n}(k)\end{array}\right]$

with $\mathbf{p}^{n}(k), \mathbf{v}^{n}(k), \mathbf{q}^{n}(k)$ representing position, velocity, and quaternion respectively at discrete time $\mathrm{k}, \Delta t$ being the time for update interval, $\mathbf{q}^{n}(k)$ being the attitude quaternion with $\otimes$ for the quaternion multiplication, and $\triangle \mathbf{q}^{n}(k)$ being a delta quaternion computed from gyroscope readings during the attitude update interval.

The wind velocity and feature map are maintained outside the SLAM filter and are treated as external databases.

\section{BAYESIAN MODEL FOR INDIRECT SLAM}

The airborne SLAM problem with unknown wind velocity and unknown map position needs to compute the probability density function given all observations and control inputs up to current time $k$. In indirect SLAM, only the probability function for the error variables are of interest:

$$
p\left(\delta \mathbf{x}_{k}, \delta \mathbf{m}, \delta \mathbf{w} \mid \delta \mathbf{Z}_{k}, \mathbf{U}_{k}\right)
$$

where,

$\delta \mathbf{x}_{k}$ is a vehicle state such as position, velocity and attitude $\delta \mathbf{m}$ is a stationary map position

$\delta \mathbf{w}$ is a wind velocity

$\delta \mathbf{Z}_{k}$ is a feature and air velocity observation:

$$
\delta \mathbf{Z}_{k}=\left\{\delta \mathbf{Z}_{k}^{f}, \delta \mathbf{Z}_{k}^{a}\right\}=\left\{z_{0}^{f}, z_{1}^{f}, \cdots, z_{k}^{f}, z_{0}^{a}, z_{1}^{a}, \cdots, z_{a}^{f}\right\}
$$

$\mathbf{U}_{k}$ is an inertial sensor observation:

$$
\mathbf{U}_{k}=\left\{u_{0}, u_{1}, \cdots, u_{k}\right\}
$$

From the Bayes theorem, a posterior distribution can be obtained from the likelihood and predicted distribution,

$$
\begin{aligned}
p( & \left.\delta \mathbf{x}_{k}, \delta \mathbf{m}, \delta \mathbf{w} \mid \delta \mathbf{Z}_{k}, \mathbf{U}_{k}\right) \\
\propto & p\left(\delta \mathbf{z}_{k}^{f}, \delta \mathbf{z}_{k}^{a} \mid \delta \mathbf{x}_{k}, \delta \mathbf{m}, \delta \mathbf{w}\right) p\left(\delta \mathbf{x}_{k}, \delta \mathbf{m}, \delta \mathbf{w} \mid \delta \mathbf{Z}_{k-1}, \mathbf{U}_{k}\right) \\
= & p\left(\delta \mathbf{z}_{k}^{f} \mid \delta \mathbf{x}_{k}, \delta \mathbf{m}, \delta \mathbf{w}\right) p\left(\delta \mathbf{z}_{k}^{a} \mid \delta \mathbf{x}_{k}, \delta \mathbf{m}, \delta \mathbf{w}\right) \\
& \times p\left(\delta \mathbf{x}_{k}, \delta \mathbf{m}, \delta \mathbf{w} \mid \delta \mathbf{Z}_{k-1}, \mathbf{U}_{k}\right) \\
= & \underbrace{}_{\text {low feature obs-update }}\left(\delta \mathbf{z}_{k}^{f} \mid \delta \mathbf{x}_{k}, \delta \mathbf{m}\right) \times \underbrace{p\left(\delta \mathbf{z}_{k}^{a} \mid \delta \mathbf{x}_{k}, \delta \mathbf{w}, \delta \mathbf{Z}_{k-1}, \mathbf{U}_{k}\right)}_{\text {high velocity-obs update }}
\end{aligned}
$$

where the property of conditional independence between the feature and air velocity observation is used and it is assumed that the feature observation is synchronised to velocity observation. Thus the periodic velocity based inner SLAM loop can be separated from the aperiodic feature based outer SLAM loop which was shown in Fig. 1.

\section{INDIRECT SLAM IMPLEMENTATION}

In this paper, a Kalman filter is used to implement the indirect airborne SLAM by using the linearised SLAM error model [5].

The linearised SLAM system in discrete time can be written as

$$
\begin{aligned}
\delta \mathbf{x}(k+1) & =\mathbf{F}(k) \delta \mathbf{x}(k)+\mathbf{G}(k) \mathbf{w}(k) \\
\delta \mathbf{z}(k) & =\mathbf{H}(k) \delta \mathbf{x}(k)+\mathbf{v}(k),
\end{aligned}
$$

where $\delta \mathbf{x}$ is the error state vector, $\mathbf{F}(k)$ is the time-varying state transition matrix, $\mathbf{G}(k)$ is the system noise input matrix and $\mathbf{w}(k)$ is the system noise vector which represents any unmodelled instrument errors with noise strength $\mathbf{Q}(k) . \mathbf{H}(k)$ is 
the linearised observation Jacobian and $\mathbf{v}(k)$ is the observation noise with noise strength matrix $\mathbf{R}(k)$. The error observations are generated by subtracting the measured quantity $\tilde{\mathbf{z}}(k)$ from the INS predicted quantity $\hat{\mathbf{z}}(k)$,

$$
\delta \mathbf{z}(k)=\hat{\mathbf{z}}(k)-\tilde{\mathbf{z}}(k) .
$$

The state consists of the errors in INS, wind velocity, and map:

$$
\delta \mathbf{x}(k)=\left[\begin{array}{lll}
\delta \mathbf{x}_{\text {ins }}(k) & \delta \mathbf{v}_{\text {wind }}(k) & \delta \mathbf{x}_{\text {map }}(k)
\end{array}\right]^{T} .
$$

The error state of INS, $\delta \mathbf{x}_{\text {ins }}(k)$, comprises the errors in the INS indicated position, velocity and attitude expressed in the navigation frame which is defined along north, east and down axes at the current vehicle position:

$$
\delta \mathbf{x}_{\text {ins }}(k)=\left[\begin{array}{lll}
\delta \mathbf{p}^{n}(k) & \delta \mathbf{v}^{n}(k) & \delta \boldsymbol{\psi}^{n}(k)
\end{array}\right]^{T} .
$$

The error state vector of the wind velocity is also defined in the navigation frame. Although a more sophisticated model can be incorporated [6], a constant wind model is used in this paper for an initial demonstration. The error state of map, $\delta \mathbf{x}_{\text {map }}(k)$, comprises the errors in $3 \mathrm{D}$ feature positions in the navigation frame. A stationary model is used and the size of the state is dynamically augmented with the new feature.

The resulting detailed discrete state transition matrix $\mathbf{F}(k)$ and the noise transfer function $\mathbf{G}(k)$ can be found in [4].

\section{A. Feature Observation}

The on-board feature observation sensor provides range $(\rho)$, bearing $(\varphi)$, and elevation $(\vartheta)$ between the vehicle and feature. The nonlinear observation equation relates these observations to the state through the Cartesian to polar transformation, and the navigation to sensor frame transformation [4]. The sensor frame is assumed to be aligned to the body frame. The observation can then be predicted from the relative position vector between the feature and vehicle:

$$
\begin{aligned}
\hat{\mathbf{z}}_{\text {feature }}^{s}(k) & =\left[\begin{array}{lll}
\hat{\rho} & \hat{\varphi} & \hat{\vartheta}
\end{array}\right]^{T} \\
& =\left[\begin{array}{c}
\sqrt{\hat{x}^{2}+\hat{y}^{2}+\hat{z}^{2}} \\
\arctan (\hat{y} / \hat{x}) \\
\arcsin (\hat{z} / \hat{\rho})
\end{array}\right]
\end{aligned}
$$

where the relative position vector is

$$
\left[\begin{array}{lll}
\hat{x} & \hat{y} & \hat{z}
\end{array}\right]^{T}=\mathbf{C}_{n}^{b}\left[\hat{\mathbf{m}}^{n}-\hat{\mathbf{p}}^{n}\right]
$$

The linearised $\mathbf{H}(k)$ with respect to the current vehicle and map state can be derived from the Jacobian of these nonlinear observation equations,

$\mathbf{H}(k)=\left[\begin{array}{ccc|c|ccc}\frac{\partial \rho}{\partial \mathbf{p}^{n}} & \frac{\partial \rho}{\partial \mathbf{v}^{n}} & \frac{\partial \rho}{\partial \psi^{n}} & 0_{1 \times 3} & \cdots & -\frac{\partial \rho}{\partial \mathbf{p}^{n}} & \cdots \\ \frac{\partial \varphi}{\partial \mathbf{p}^{n}} & \frac{\partial \varphi}{\partial \mathbf{v}^{n}} & \frac{\partial \varphi}{\partial \psi^{n}} & 0_{1 \times 3} & \cdots & -\frac{\partial \varphi}{\partial \mathbf{p}^{n}} & \cdots \\ \frac{\partial \vartheta}{\partial \mathbf{p}^{n}} & \frac{\partial \vartheta}{\partial \mathbf{v}^{n}} & \frac{\partial \vartheta}{\partial \boldsymbol{\psi}^{n}} & 0_{1 \times 3} & \cdots & -\frac{\partial \vartheta}{\partial \mathbf{p}^{n}} & \cdots\end{array}\right]$

with corresponding sensor noise variance

$$
\mathbf{R}(k)=\left[\begin{array}{ccc}
\sigma_{\rho}^{2} & 0 & 0 \\
0 & \sigma_{\varphi}^{2} & 0 \\
0 & 0 & \sigma_{\vartheta}^{2}
\end{array}\right] .
$$

\section{B. Air-Data Observation}

The on-board air-data system can deliver air speed $(V)$, angle of attack $(\alpha)$, and side slip angle $(\beta)$ from the pitot tube and alpha/beta vanes [6]. Fig. 2 describes the typical pitot system and the geometry of the aerodynamic angles. The pitot tube is typically installed on the front nose or wing tip in pushprop type vehicles.

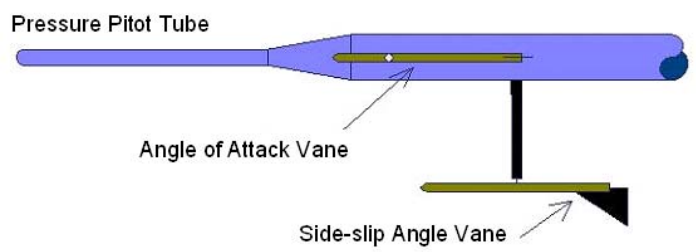

(a)

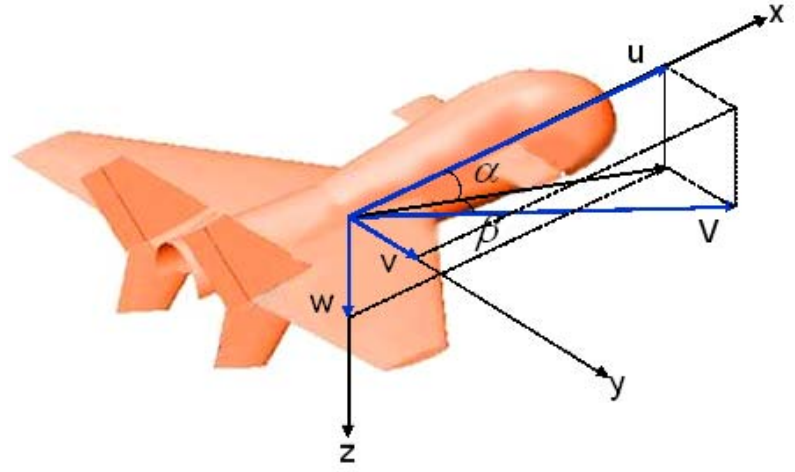

(b)

Fig. 2. a) A pitot tube for pressure measurement with alpha/beta vanes for aerodynamic angles. b) The geometry of the air velocity vector with the aerodynamic angles.

The pressure measurements can be converted into the airspeed which is the vehicle velocity with respect to the ambient air mass. By using the observation geometry, the air velocity vector in the body frame $\mathbf{v}_{a i r}^{b}$ can be constructed,

$$
\begin{aligned}
\mathbf{v}_{\text {air }}^{b} & =\left[\begin{array}{lll}
u & v & w
\end{array}\right]^{T} \\
& =\left[\begin{array}{c}
V \cos (\alpha) \cos (\beta) \\
V \sin (\beta) \\
V \sin (\alpha) \cos (\beta)
\end{array}\right] .
\end{aligned}
$$

By taking an inverse of this equation, the air velocity observation $(\hat{V}, \hat{\alpha}, \hat{\beta})$ can be predicted from the relative air velocity in the body frame $(\hat{u}, \hat{v}, \hat{w})$,

$$
\begin{aligned}
\hat{\mathbf{z}}_{a i r}^{b} & =\left[\begin{array}{lll}
\hat{V} & \hat{\alpha} & \hat{\beta}
\end{array}\right]^{T} \\
& =\left[\begin{array}{c}
\sqrt{\hat{u}^{2}+\hat{v}^{2}+\hat{w}^{2}} \\
\arctan (\hat{w} / \hat{u}) \\
\arcsin (\hat{v} / \hat{V})
\end{array}\right],
\end{aligned}
$$

where, the relative air velocity is predicted by subtracting the estimated wind velocity from the estimated INS velocity, and by transforming it into the body frame,

$$
\left[\begin{array}{lll}
\hat{u} & \hat{v} & \hat{w}
\end{array}\right]^{T}=\mathbf{C}_{n}^{b}\left[\hat{\mathbf{v}}_{i n s}^{n}-\hat{\mathbf{v}}_{w i n d}^{n}\right] .
$$


The linearised observation model is obtained by taking the Jacobian of these nonlinear equations,

$$
\begin{gathered}
\mathbf{H}(k)=\left[\begin{array}{ccc|c|c}
0_{1 \times 3} & \frac{\partial V}{\partial \mathbf{v}^{n}} & \frac{\partial V}{\partial \boldsymbol{\psi}^{n}} & -\frac{\partial V}{\partial \mathbf{v}^{n}} & 0_{1 \times 3 m} \\
0_{1 \times 3} & \frac{\partial \alpha}{\partial \mathbf{v}^{n}} & \frac{\partial \alpha}{\partial \boldsymbol{\psi}^{n}} & -\frac{\partial \alpha}{\partial \mathbf{v}^{n}} & 0_{1 \times 3 m} \\
0_{1 \times 3} & \frac{\partial \beta}{\partial \mathbf{v}^{n}} & \frac{\partial \beta}{\partial \boldsymbol{\psi}^{n}} & -\frac{\partial \beta}{\partial \mathbf{v}^{n}} & 0_{1 \times 3 m}
\end{array}\right] \\
\mathbf{R}(k)=\left[\begin{array}{ccc}
\sigma_{V}^{2} & 0 & 0 \\
0 & \sigma_{\alpha}^{2} & 0 \\
0 & 0 & \sigma_{\beta}^{2}
\end{array}\right]
\end{gathered}
$$

Note the similarity between the feature and air velocity observation models due to the nature of the three dimensional vector observations. Hence minimal software modification is required.

\section{Error Feedback Correction}

The corrected position, $\mathbf{p}^{n}(k \mid k)$, and velocity, $\mathbf{v}^{n}(k \mid k)$, are computed by subtracting the estimated INS errors from the current INS outputs:

$$
\begin{aligned}
& \mathbf{p}^{n}(k \mid k)=\mathbf{p}^{n}(k)-\delta \mathbf{p}^{n}(k \mid k) \\
& \mathbf{v}^{n}(k \mid k)=\mathbf{v}^{n}(k)-\delta \mathbf{v}^{n}(k \mid k) .
\end{aligned}
$$

The corrected quaternion, $\mathbf{q}(k \mid k)$, is obtained by premultiplying the error quaternion to the current quaternion:

$$
\begin{aligned}
\mathbf{q}(k \mid k) & =\delta \mathbf{q}(k \mid k) \otimes \mathbf{q}(k) \\
\delta \mathbf{q}(k \mid k) & \approx\left[\begin{array}{llll}
1 & \delta \psi_{x} / 2 & \delta \psi_{y} / 2 & \delta \psi_{z} / 2
\end{array}\right]^{T}
\end{aligned}
$$

The wind velocity is directly corrected by subtracting the estimated wind velocity error from the current wind velocity:

$$
\mathbf{v}_{w}^{n}(k \mid k)=\mathbf{v}_{w}^{n}(k)-\delta \mathbf{v}_{w}^{n}(k \mid k)
$$

The map positions are also corrected by subtracting the estimated map position errors from the current positions:

$$
\mathbf{x}_{m}^{n}(k \mid k)=\mathbf{x}_{m}^{n}(k)-\delta \mathbf{x}_{m}^{n}(k \mid k) .
$$

\section{Simulation Description}

A computer simulation is performed to verify the robustness of the proposed method. The flight scenario is the vehicle undertaking two racehorse tracks approximately $100 \mathrm{~m}$ above the ground. The flight time is 340 seconds and the average flight speed is $40 \mathrm{~m} / \mathrm{s}$. There are 40 features placed on the ground.

The wind is a prior unknown to the vehicle and has a magnitude of $5.0 \mathrm{~m} / \mathrm{s}$ with a southerly direction. Inside the SLAM filter, this unknown wind velocity is estimated from the relative velocity observation. The air data sensors run at $20 \mathrm{~Hz}$. The airspeed has a noise value of $1 \mathrm{~m} / \mathrm{s}$ and the alpha vane and beta vane angles have noise values of $2^{\circ}$ respectively.

A low-cost IMU is simulated with a sampling rate of $400 \mathrm{~Hz}$ and has an average noise value of $0.5 \mathrm{~m} / \mathrm{s}^{2}$ for the accelerometers and $0.5^{\circ} / \mathrm{s}$ for the gyros. The vision sensor is used to measure the range, azimuth and elevation angles to each feature on the ground. The vision sensors run at 10 frames/s and have a horizontal field of view of $40^{\circ}$ and a vertical field of view of $30^{\circ}$. In the simulation we assume known data association of the map features.

\section{Vi. Simulation Results}

Fig. 3 compares the horizontal position results between unaided inertial, velocity based SLAM and velocity/featurebased, or multi-loop, SLAM. The velocity based SLAM can limit the error growth of the velocity and attitude but not the position error. By fusing the feature observation the multi-loop SLAM can estimate the position error.

Fig. 4 compares the $1 \sigma$ covariances between the unaided inertial, velocity based SLAM, and multi-loop SLAM. The position and velocity along the north axis are plotted and the attitude along the yaw axis is shown. It can be observed that the velocity and attitude performances are improved in the velocity based SLAM loop. It however shows that the position error increases without bound. The feature observation provides additional position information to the vehicle state and improves overall performance.

Fig. 5 shows the Monte-Carlo analysis results for the wind velocity and attitude estimates in the multi-loop SLAM. The Monte-Carlo method is used to see the statistical consistency of the SLAM filter particulary during the loop closure. Fig. 5 (a) shows ten runs along the north axis with $3 \sigma$ bound. The true wind velocity was $[-5,0,0]^{T}(\mathrm{~m} / \mathrm{s})$. It can be observed that the unknown wind vector can be estimated consistently during the first loop closure which is around 70 seconds. An interesting thing is the loop closure also improves the wind velocity estimate. This is because the INS has developed a cross-correlation between the position and velocity during the flight and any position improvement affects the velocity as well. Fig. 5(b) and (c) show Monte-Carlo results for the roll and yaw angles during the first loop closure. Angles from ten runs are plotted with $3 \sigma$ bounds respectively. These show consistent angle estimates of the vehicle within the bounds. This is important in airborne SLAM since the attitude estimate can now be less susceptible to the availability and quality of the ground feature observations.

These results show that the multi-loop SLAM has a capability to estimate the unknown wind velocity reliably and more importantly the consistency of SLAM can be significantly improved when it tries to close the loop.

\section{CONCLUSIONS}

This paper presented a multi-loop SLAM structure to improve the consistency of airborne SLAM. The inner SLAM loop was configured by using the periodic air-velocity update and the outer SLAM loop by the aperiodic feature update. The unknown wind velocity was augmented and estimated from the inner SLAM loop to stabilise the INS velocity and attitude. The feature observation update was still required to estimate the error growth in the INS position. The Monte-Carlo analysis verified that multi-loop SLAM is statistically consistent during the loop closure. This result can also be directly applicable to the underwater inertial SLAM problem. That is, the relative velocity measurement with respect to the underwater current can be used to stabilise SLAM. Future work is to build a geographical wind map in multi-loop SLAM to account for the terrain variations in near-Earth flight. 


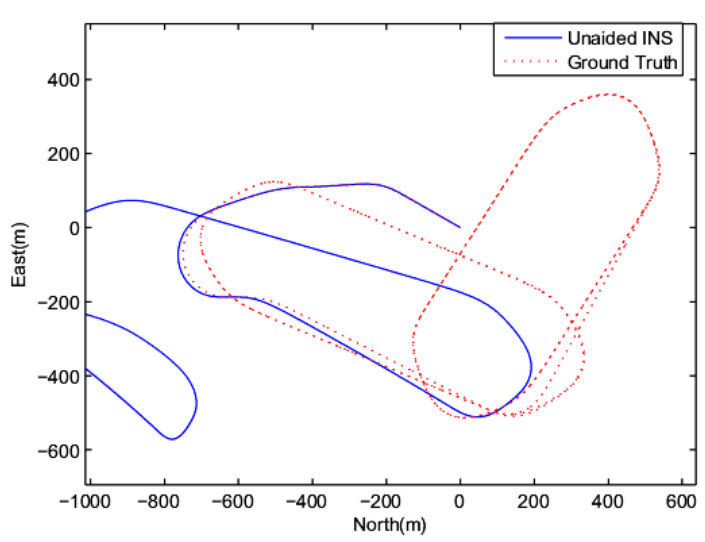

(a)

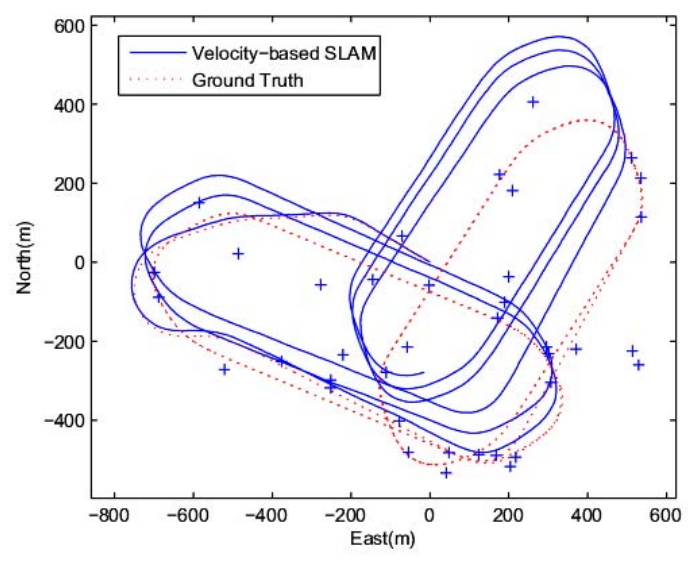

(b)

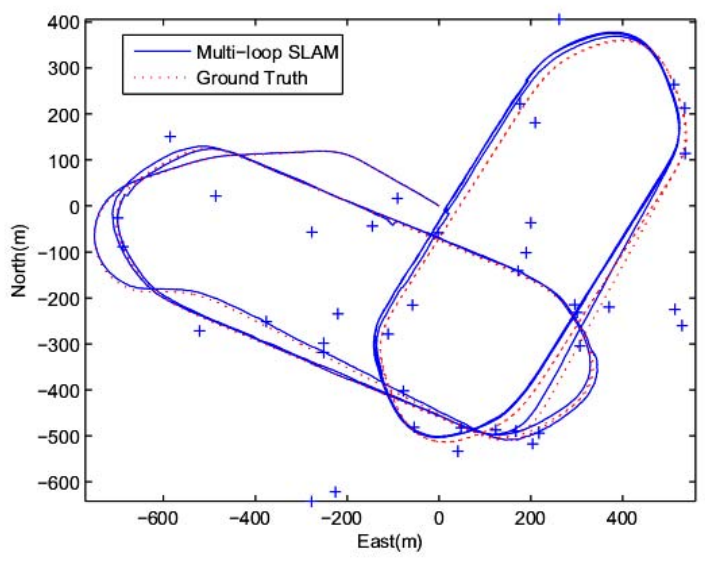

(c)

Fig. 3. A comparison of $2 \mathrm{D}$ position results between unaided inertial, velocity based SLAM, and velocity/feature based SLAM. The velocity based SLAM improves the velocity and attitude estimates but the position still drifts. The multi-loop SLAM overcomes this and improves the filter stability.

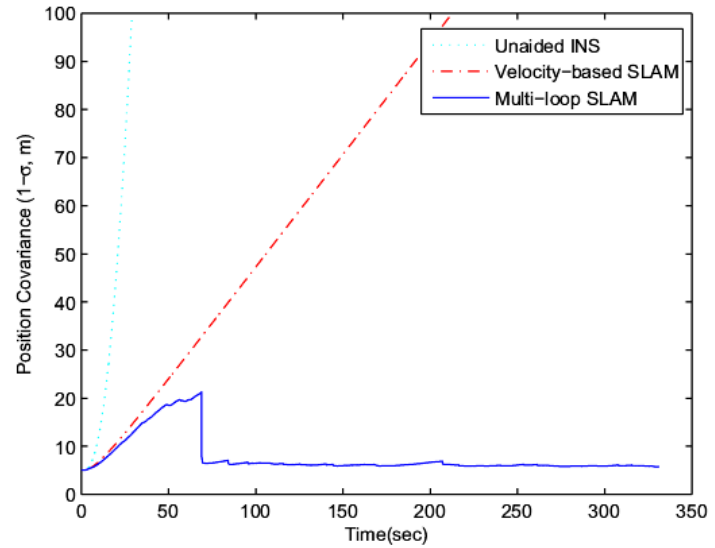

(a)

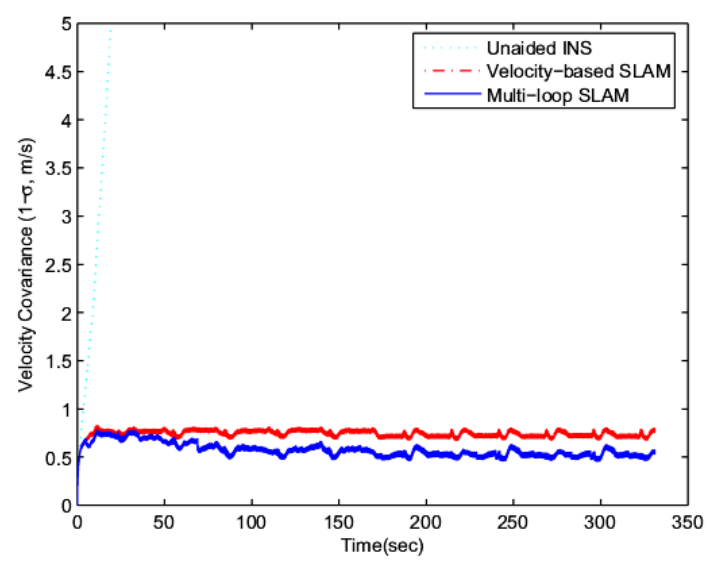

(b)

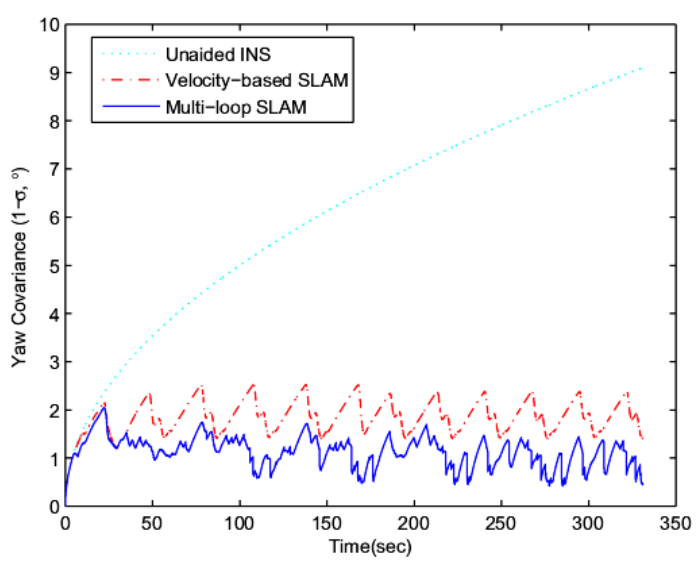

(c)

Fig. 4. Comparisons of error covariances between unaided inertial, velocity based SLAM, and velocity/feature-based SLAM. The multi-loop SLAM improves the overall performances in the position, velocity and attitude. 


\section{REFERENCES}

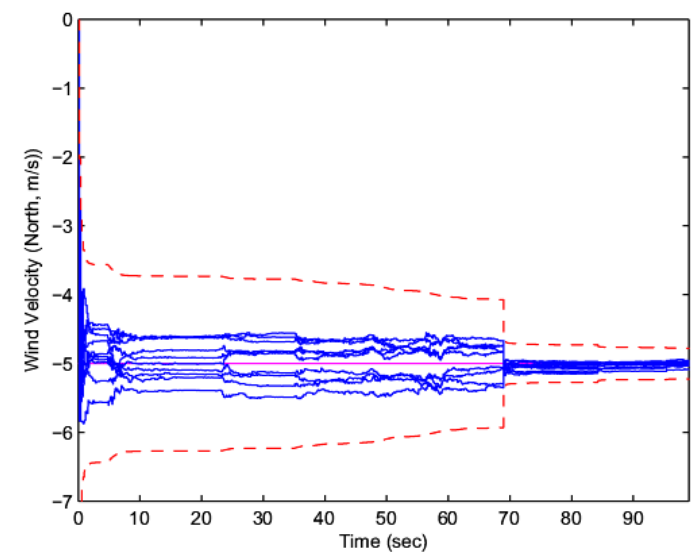

(a)

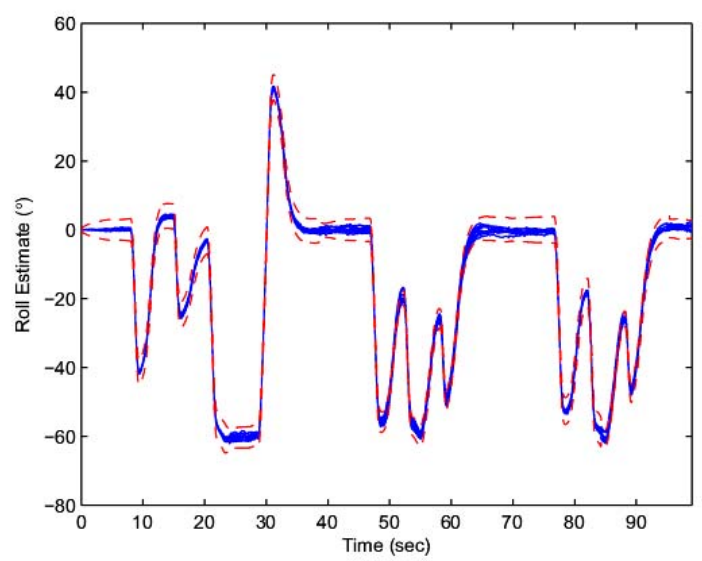

(b)

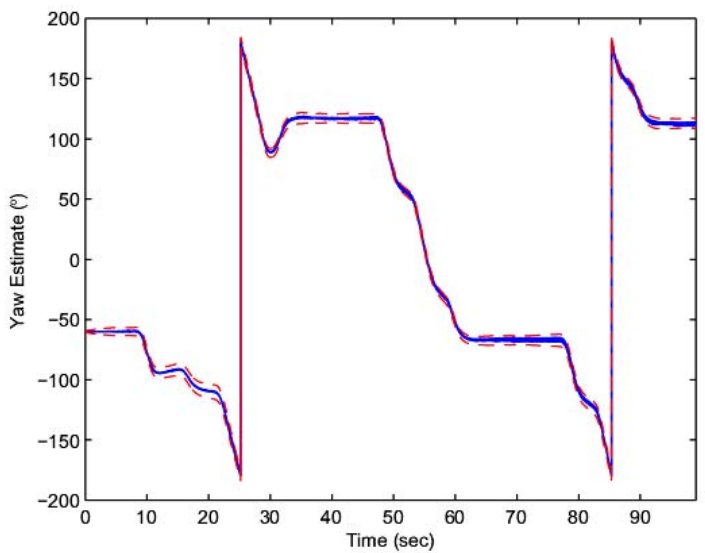

(c)

Fig. 5. a) A wind velocity estimation result in multi-loop SLAM from the Monte-Carlo analysis. The true wind velocity is $5 \mathrm{~m} / \mathrm{s}$ southward. The estimated wind velocities from ten runs are plotted with 3- $\sigma$ uncertainty. Monte-Carlo analysis results for b) roll and c) yaw angles with 3- $\sigma$ bounds showing a consistent loop closure.
[1] S. Williams and I. Mahon, "Simultaneous localization and mapping on the Great Barrier Reef," in IEEE International Conference on Robotics and Automation, 2004.

[2] A. Nuchter, S. H., L. K., H. J., and T. S., "6D SLAM with an Application in Autonomous Mine Mapping," IEEE Transactions on Robotics and Automation, pp. 1998-2003, 2004.

[3] R. Chatila, M.Devy, and J. Tardos, SLAM 2004 Summer School: Lecture Notes. LAAS, Toulouse, France, 2004.

[4] J. Kim, "Autonomous Navigation for Airborne Applications," $\mathrm{PhD}$ thesis, Australian Centre for Field Robotics, The University of Sydney, 2004.

[5] J. Kim and S. Sukkarieh, "Improving the Real-Time Efficiency of Inertial SLAM and Understanding its Observability," in IEEE/RSJ International Conference on Intelligent Robots and Systems, Sendai, Japan, 2004.

[6] B. Stevens and F. Lewis, Aircraft Control and Simulation. John Wiley and Sons, Inc, 1992. 